\title{
ON THE NUMERICAL INTEGRATION OF WALSH SERIES BY NUMBER-THEORETIC METHODS
}

\author{
GERHARD LARCHER AND CLAUDIA TRAUNFELLNER
}

\begin{abstract}
In analogy to the theory of good lattice points for the numerical integration of rapidly converging Fourier series, a theory for the fast numerical integration of Walsh series is developed. The basis for this theory is provided by a class of very well-distributed point sets in the $s$-dimensional unit cube, the so-called $(t, m, s)$-nets.
\end{abstract}

\section{INTRODUCTION}

One of the main branches in the theory of numerical integration by numbertheoretic methods is the theory of good lattice points. Good lattice point methods were introduced independently by Hlawka [4] and Korobov [6], and are an excellent tool for the numerical integration of functions which are representable by, in a certain sense, rapidly converging Fourier series. The basic principle is the following:

Let $f: \mathbb{R}^{s} \rightarrow \mathbb{R}$ be periodic with period one in each variable. Let

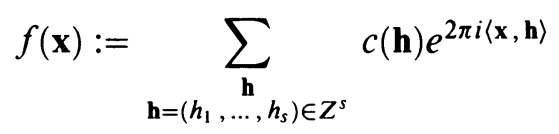

for all $\mathbf{x} \in \mathbb{R}^{s}$, and for an $\alpha>1$ and a $c>0$ let

$$
|c(\mathbf{h})|<c\left(\bar{h}_{1} \cdots \bar{h}_{s}\right)^{-\alpha} \quad \text { with } \bar{h}_{i}:=\max \left(1,\left|h_{i}\right|\right),
$$

for all $\mathbf{h}$. We then say that $f$ belongs to the class $E_{s}^{\alpha}(c)$. Under these assumptions we have:

There is a constant $c^{\prime}=c^{\prime}(c, \alpha, s)$ such that for every $N \in \mathbb{N}$ there exists a "good lattice point" $\mathbf{g}:=\left(g_{1}, \ldots, g_{s}\right) \in \mathbb{Z}^{s}$ so that

$$
\left|\int_{[0,1)^{s}} f(x) d x-\frac{1}{N} \sum_{k=1}^{N} f\left(\frac{k}{N} \cdot \mathbf{g}\right)\right| \leq c^{\prime} \frac{(\log N)^{\alpha s}}{N^{\alpha}} .
$$

$($ See $[4,7,12]$.

For the case $N=p$ prime, the above result was improved by Bahvalov [1]. In this case one gets an error estimate of the form

$$
c^{\prime} \frac{(\log N)^{\alpha(s-1)}}{N^{\alpha}} \text {. }
$$

Received by the editor July 27, 1992 and, in revised form, February 19, 1993.

1991 Mathematics Subject Classification. Primary 65D30, 11K45. 
For composite $N$ the best result known until quite recently was established by Niederreiter [17] with an error estimate of the form

$$
c^{\prime \prime} \frac{(\log N)^{\alpha(s-1)+1}}{N^{\alpha}}\left(\frac{N}{\phi(N)}\right)^{(\alpha-1)(s-1)},
$$

where $\phi$ is Euler's totient function.

The method of good lattice points has the great advantage that the smoothness of the function $f$ is highly beneficial, and it gives, especially in higher dimension, essentially better results than classical integration methods such as those based on a uniform lattice. Thus, for example, if we take the point set

$$
\left(\frac{k_{1}}{n}, \ldots, \frac{k_{s}}{n}\right), \quad k_{i}=1, \ldots, n, i=1, \ldots, s, N=n^{s}
$$

for the numerical integration, then we only get an error estimate of the form $c N^{-\alpha / s}$. Increasing the dimension $s$, therefore, contributes in a much more essential way to an increase in the integration error.

However, especially for dimension $s \geq 3$, it turned out to be a challenge to give fast algorithms for finding good lattice points $\mathbf{g}$ to a given modulus $N$. In dimension $s=2$, with the help of the continued fraction algorithm, it is possible to give effective methods for constructing good lattice points. (See, for example, [7] and [13].) For dimensions $s \geq 3$ one essentially has to rely on tabulated values.

In recent years, in connection with image and signal processing, the importance of Walsh series, respectively of functions which are representable by Walsh series, has steadily grown. (See for example [10].) First attempts in the direction of giving good methods for the numerical integration of such Walsh series were already made by Sobol. (See [19], [20]; he indeed works with Haar functions.) But only by a systematic development of the theory of nets by Niederreiter (such nets will in the following play the role of the good lattice point sequences), which has turned out to be enormously fruitful in the theory of uniform distribution (see [14] and [15]), it became possible, first:

to give results analogous to those in the theory of good lattice points, now concerning the integration of Walsh series (this is the task of $\S 2$ of the paper), and furthermore:

to even give, with regard to such things like effectivity, constructivity of methods, and quality of the error estimates, more convincing results than in the classical good lattice point theory $(\S 3)$.

In the last section the methods developed will be applied to concrete examples.

\section{NUMERICAL INTEGRATION OF WALSH SERIES}

Definition. Let $b \geq 2$ be an integer and $w:=e^{2 \pi i / b}$. The Rademacher functions to base $b$ are defined by

$$
{ }_{b} \phi_{0}(x)=w^{k} \text { for } k / b \leq x<(k+1) / b, k=0, \ldots, b-1,
$$

and for $n \geq 0$ by

$$
{ }_{b} \phi_{n}(x+1):={ }_{b} \phi_{n}(x):={ }_{b} \phi_{0}\left(b^{n} x\right) \text {. }
$$

Definition. The Walsh functions to base $b$ are defined by

$$
{ }_{b} \mathbf{w a l}_{0}(x)=1
$$


and if $n=a_{1} b^{n_{1}}+\cdots+a_{m} b^{n_{m}}$ with $0 \leq a_{i}<b$ and $n_{1}>n_{2}>\cdots$ by

$$
{ }_{b} \operatorname{wal}_{n}(x)={ }_{b} \phi_{n_{1}}^{a_{1}}(x) \cdots{ }_{b} \phi_{n_{m}}^{a_{m}}(x) .
$$

For dimension $s \geq 2$ and $k_{1}, \ldots, k_{s} \geq 0$ we define

$$
{ }_{b} \mathrm{wal}_{k_{1}, \ldots, k_{s}}\left(x_{1}, \ldots, x_{s}\right)={ }_{b} \mathrm{wal}_{k_{1}}\left(x_{1}\right) \cdots_{b} \mathbf{w a l}_{k_{s}}\left(x_{s}\right) \text {. }
$$

The system of functions $W=\left\{{ }_{b} \mathrm{wal}_{k_{1}, \ldots, k_{s}} \mid k_{i} \geq 0, i=1, \ldots, s\right\}$, is orthonormal and complete in $L^{2}\left([0,1)^{s}\right)$. (See [2] and [10].)

Definition. For given base $b \geq 2$ and for $\alpha>1$ and $c>0$ let ${ }_{b} \bar{E}_{s}^{\alpha}(c)$ be the class of all $f: \mathbb{R}^{s} \rightarrow \mathbb{R}$ with

$$
f(\mathbf{x})=\sum_{k_{1}, \ldots, k_{s}=0}^{\infty} \bar{f}\left(k_{1}, \ldots, k_{s}\right)_{b} \text { wal }_{k_{1}, \ldots, k_{s}}(\mathbf{x})
$$

for all $\mathbf{x} \in[0,1)^{s}$ and with $\left|\bar{f}\left(k_{1}, \ldots, k_{s}\right)\right| \leq c\left(\bar{k}_{1} \cdots \bar{k}_{s}\right)^{-\alpha}$ for all $\mathbf{k}:=$ $\left(k_{1}, \ldots, k_{s}\right)$.

(In the following we write wal instead of ${ }_{b}$ wal, and $\bar{E}_{s}^{\alpha}$ instead of ${ }_{b} \bar{E}_{s}^{\alpha}$ if it is clear what base $b$ is meant.)

Definition. For an integer $b \geq 2$ an elementary interval to base $b$ is defined to be an interval of the form

$$
\prod_{i=1}^{s}\left[a^{(i)} b^{-d_{i}},\left(a^{(i)}+1\right) b^{-d_{i}}\right)
$$

with integers $d_{i}, a^{(i)} \geq 0$ and $a^{(i)}<b^{d_{i}}$ for $1 \leq i \leq s$.

Definition. Let $b \geq 2$ and $0 \leq t \leq m$ be integers. A $(t, m, s)$-net to base $b$ is a set of $b^{m}$ points in $[0,1)^{s}$ having the property that in every elementary interval to base $b$ of volume $b^{t-m}$ there are exactly $b^{t}$ points of the set.

(For the last two definitions see [14].)

First we show:

Theorem 1. For a given base $b \geq 2$, let $f \in \bar{E}_{s}^{\alpha}(c), \mathbf{x}_{1}, \ldots, \mathbf{x}_{N} \in[0,1)^{s}$, and

$$
R_{N}(f):=\left|\int_{[0,1)^{s}} f(\mathbf{x}) d \mathbf{x}-\frac{1}{N} \sum_{k=1}^{N} f\left(\mathbf{x}_{k}\right)\right| .
$$

(a) If $N=b^{s n}$ and $\mathbf{x}_{1}, \ldots, \mathbf{x}_{N}$ is the uniform lattice, then

$$
R_{N}(f) \leq c\left(\left(1+\frac{\zeta(\alpha)}{b^{n \alpha}}\right)^{s}-1\right) \leq c^{\prime}(s, b, \alpha, c) N^{-\alpha / s} .
$$

( $\zeta$ is Riemann's zeta-function.)

(b) If $\mathbf{x}_{1}, \ldots, \mathbf{x}_{N}$ is a $(t, m, s)$-net to base $b$, then

$$
R_{N}(f) \leq c^{\prime \prime}(s, b, \alpha, c) b^{(\alpha-1) t} \frac{(\log N)^{s-1}}{N^{\alpha-1}} .
$$

(An estimate for $c^{\prime \prime}$ can easily be given by the proof of (b).)

For the proof we start with four easy lemmas. 
Lemma 1. We have $R_{N}(f)=\left|\frac{1}{N} \sum_{\mathbf{k} \neq \mathbf{0}} \bar{f}(\mathbf{k}) \cdot S_{N}(\mathbf{k})\right|$ with $S_{N}(\mathbf{k})=\sum_{j=1}^{N} \mathbf{w a l}_{\mathbf{k}}\left(\mathbf{x}_{j}\right)$. Proof. Inserting the absolutely converging Walsh series in the definition of $R_{N}(f)$ gives the result.

Lemma 2. For $f \in \bar{E}_{s}^{\alpha}(c)$ we have

$$
R_{N}(f) \leq \frac{c}{N} \sum_{\mathbf{k} \neq \mathbf{0}} \frac{\left|S_{N}(\mathbf{k})\right|}{\left(\bar{k}_{1} \cdots \bar{k}_{s}\right)^{\alpha}} .
$$

Proof. Follows immediately from Lemma 1.

Lemma 3. (a) If $k=a b^{n}+k^{\prime}$, then $\operatorname{wal}_{k}\left(l / b^{n}\right)=\operatorname{wal}_{k^{\prime}}\left(l / b^{n}\right)$ for all $l \in \mathbb{N}$.

(b) If $k=b k^{\prime}+r$ with $0 \leq r<b$, then $\operatorname{wal}_{k}(x)=$ wal $_{k^{\prime}}(b x) w^{r k(x)}$ with $k(x) / b \leq x<(k(x)+1) / b$.

(c)

$$
\sum_{l_{1}, \ldots, l_{s}=0}^{b^{n}-1} \operatorname{wal}_{\mathbf{k}}\left(\frac{l_{1}}{b^{n}}, \ldots, \frac{l_{s}}{b^{n}}\right)= \begin{cases}b^{n s} & \text { if } b^{n} \mid k_{i} \text { for all } i=1, \ldots, s, \\ 0 & \text { otherwise. }\end{cases}
$$

Proof. Assertions (a) and (b) immediately follow from the definition of Walsh functions, and (c) follows from

$$
\begin{aligned}
& \sum_{l=0}^{b^{n}-1} \mathrm{wal}_{k}\left(l / b^{n}\right)=\sum_{l=0}^{b^{n}-1} \mathrm{wal}_{k^{\prime}}\left(l / b^{n-1}\right) w^{r k\left(l / b^{n}\right)} \\
& =\sum_{k=0}^{b-1} w^{r k} \sum_{l=0}^{b^{n-1}-1} \operatorname{wal}_{k^{\prime}}\left(l / b^{n-1}\right)=0 \quad \text { if } r \neq 0 .
\end{aligned}
$$

(Here we put $k=b k^{\prime}+r$ as in (b).)

Lemma 4. Let $N=b^{m}$ and let $\mathbf{x}_{n}, n=1, \ldots, N$, be a $(t, m, s)$-net to base $b$. Then we have: if $\mathbf{k}:=\left(k_{1}, \ldots, k_{s}\right) \neq \mathbf{0}$ is such that there exist integers $d_{i} \geq 0$ with $k_{i}<b^{d_{i}}$ for $i=1, \ldots, s$ and $d_{1}+\cdots+d_{s}=m-t$, then $S_{N}(\mathbf{k})=0$.

Proof. Let $d_{1}, \ldots, d_{s}$ with the above property be given. We divide $[0,1)^{s}$ in $b^{m-t}$ intervals of the form

$$
E=\prod_{i=1}^{s}\left[a_{i} b^{-d_{i}},\left(a_{i}+1\right) b^{-d_{i}}\right), \quad a_{i}=0, \ldots, b^{d_{i}}-1 .
$$

Each of these intervals has volume $b^{t-m}$, and therefore in each of these intervals there are exactly $b^{t}$ points of the net. The function wal $_{k_{i}}(x)$ is constant on every interval of the form

$$
\left[a_{i} b^{-d_{i}},\left(a_{i}+1\right) b^{-d_{i}}\right),
$$

and therefore $\mathrm{wal}_{k_{1}, \ldots, k_{s}}$ is constant on each of the above intervals $E$. Therefore, we have

$$
S_{N}(\mathbf{k})=b^{t} \sum_{a_{1}, \ldots, a_{s}=0}^{b^{d_{i}-1}} \operatorname{wal}_{k_{1}}\left(a_{1} / b^{d_{1}}\right) \cdots \operatorname{wal}_{k_{s}}\left(a_{s} / b^{d_{s}}\right)=0,
$$

which follows from Lemma 3(c). 
Proof of Theorem 1. (a) From Lemma 2 and Lemma 3(c) we have

$$
\begin{aligned}
R_{N}(f) & \leq \frac{c}{N} \sum_{r=1}^{s}\left(\begin{array}{l}
s \\
r
\end{array}\right) \sum_{l_{1}, \ldots, l_{r}=1}^{\infty} b^{-n r \alpha} b^{n s}\left(l_{1} \cdots l_{r}\right)^{-\alpha} \\
& =c \sum_{r=1}^{s}\left(\begin{array}{l}
s \\
r
\end{array}\right) b^{-n r \alpha} \zeta^{r}(\alpha)=c\left(\left(1+\frac{\zeta(\alpha)}{b^{n \alpha}}\right)^{s}-1\right) .
\end{aligned}
$$

(b) From Lemma 2 and Lemma 4 we have

$$
R_{N}(f) \leq c \sum_{r=1}^{s}\left(\begin{array}{l}
s \\
r
\end{array}\right) \sum_{\substack{d_{1}, \ldots, d_{r}=0 \\
d_{1}+\cdots+d_{r}>m-t-r}}^{\infty}\left(\prod_{u=1}^{r} \sum_{k_{u}=b^{d_{u}}}^{b^{d_{u}+1}-1} k_{u}^{-\alpha}\right) .
$$

From $\sum_{k=A}^{\infty} k^{-\alpha} \leq c_{\alpha} A^{1-\alpha}$ we have

$$
\begin{aligned}
R_{N}(f) & \leq c \sum_{r=1}^{s}\left(\begin{array}{l}
s \\
r
\end{array}\right) c_{\alpha}^{r} \sum_{\substack{d_{1}, \ldots, d_{r}=0 \\
d_{1}+\cdots+d_{r}>m-t-r}}^{\infty} b^{(1-\alpha)\left(d_{1}+\cdots+d_{r}\right)} \\
& \leq c \sum_{r=1}^{s}\left(\begin{array}{l}
s \\
r
\end{array}\right) c_{\alpha}^{r} \sum_{\substack{i=m-t-r+1 \\
d_{1}, \ldots, d_{r}=0 \\
d_{1}+\cdots+d_{r}=i}}^{\infty} b^{(1-\alpha) i} \\
& \leq c \sum_{r=1}^{s}\left(\begin{array}{l}
s \\
r
\end{array}\right) c_{\alpha}^{r} \sum_{i=m-t-r+1}^{\infty} i^{r-1} b^{(1-\alpha) i} \\
& \leq c N^{1-\alpha} b^{(\alpha-1) t} b^{1-\alpha} \sum_{r=1}^{s}\left(\begin{array}{l}
s \\
r
\end{array}\right) c_{\alpha}^{r} b^{r(\alpha-1)}(m-t)^{r-1} \sum_{i=1}^{\infty} i^{s-1} b^{(1-\alpha) i} \\
& \leq c^{\prime \prime}(s, b, \alpha, c) b^{(\alpha-1) t} \frac{(\log N)^{s-1}}{N^{\alpha-1}} .
\end{aligned}
$$

Remark 1. The estimate in (a) is, apart from the constant $c$, best possible since if we take

$$
f(\mathbf{x}):=\sum_{k_{1}=1}^{\infty} k_{1}^{-\alpha} \mathrm{wal}_{k_{1}, 0, \ldots, 0}(\mathbf{x}),
$$

then

$$
R_{N}(f)=\frac{1}{N} \sum_{l=1}^{\infty} b^{-n \alpha} l^{-\alpha} b^{n s}=N^{-\alpha / s} \zeta(\alpha) .
$$

Remark 2. If $\alpha-1>\alpha / s$, that is, $\alpha>s(s-1)$, then with nets of any kind we get a better error estimate than for the uniform lattice. Of course, there remains the question of possibly improving the estimate. We would like to have an estimate analogous to that in the theory of good lattice points, that is, with

$$
R_{N}(f) \ll \frac{(\log N)^{\beta(s, \alpha)}}{N^{\alpha}} \text { instead of } \frac{(\log N)^{s-1}}{N^{\alpha-1}}
$$

(here, $\beta(s, \alpha)$ denotes any exponent depending at most on $s$ and $\alpha$ ) if we take the optimal case of $(0, m, s)$-nets.

But first we will show that such an improvement for arbitrary $(0, m, s)$-nets certainly is not possible: 
Theorem 2. For every $\alpha>1$ there is a $c_{\alpha}>0$ such that for all $m \in \mathbb{N}$ there exists $a(0, m, 1)$-net to base 2 and an $f \in \bar{E}_{1}^{\alpha}(1)$ such that $R_{N}(f) \geq c_{\alpha} N^{1 / 2-\alpha}$.

For the proof we first need a lemma.

Lemma 5. Let $b, k \in \mathbb{N}, b \geq 2$ and $k=b_{r-1} b^{r-1}+\cdots+b_{1} b+b_{0}$; let $x=$ $c_{1} / b+c_{2} / b^{2}+\cdots \in[0,1)$. Then

Proof. We have

$$
\operatorname{wal}_{k}(x)=w^{b_{0} c_{1}+b_{1} c_{2}+\cdots+b_{r-1} c_{r}} .
$$

$$
\operatorname{wal}_{k}(x)=\prod_{i=0}^{r-1} \phi_{0}^{b_{i}}\left(b^{i} x\right)=\prod_{i=0}^{r-1} \phi_{0}^{b_{i}}\left(\frac{c_{i+1}}{b}\right)=w^{b_{0} c_{1}+\cdots+b_{r-1} c_{r}} .
$$

Proof of Theorem 2. We consider functions of the form

$$
f(x)=2^{-(m+1) \alpha} \sum_{k=2^{m}}^{2^{m+1}-1} e_{k} \text { wal }_{k}(x) \quad \text { with } e_{k} \in\{0,1\}
$$

(therefore, $\left.f \in \bar{E}_{1}^{\alpha}(1)\right)$ and $(0, m, 1)$-nets to base 2 of the form

$$
x_{n}=\frac{a_{m}}{2}+\cdots+\frac{a_{1}}{2^{m}}+\frac{d\left(a_{m}, \ldots, a_{1}\right)}{2^{m+1}} \text { with certain } d\left(a_{m}, \ldots, a_{1}\right) \in\{0,1\},
$$

if $n=a_{m} 2^{m-1}+\cdots+a_{2} 2+a_{1}, n=0,1, \ldots, 2^{m}-1$. Then

$$
2^{\alpha} 2^{(\alpha+1) m} R_{N}(f)=\sum_{k=2^{m}}^{2^{m+1}-1} e_{k} \sum_{a_{1}, \ldots, a_{m}=0}^{1}(-1)^{d\left(a_{1}, \ldots, a_{m}\right)+a_{1} b_{m-1}+\cdots+a_{m} b_{0}}
$$

for $k=2^{m}+b_{m-1} 2^{m-1}+\cdots+b_{0}$. We consider $d$ as a function $d:\{0,1\}^{m} \rightarrow$ $\{0,1\}$. For given $d$, let $f_{d}:=\sum e_{k}$ wal $_{k}(\cdot)$ with

$$
e_{k}:=\operatorname{sgn}\left(\sum_{a_{1}, \ldots, a_{m}=0}^{1}(-1)^{d\left(a_{1}, \ldots, a_{m}\right)+a_{1} b_{m-1}+\cdots+a_{m} b_{0}}\right) \text {. }
$$

So,

$$
2^{\alpha} 2^{(\alpha+1) m} R_{N}\left(f_{d}\right)=\sum_{k=2^{m}}^{2^{m+1}-1}\left|\sum_{a_{1}, \ldots, a_{m}=0}^{1}(-1)^{d\left(a_{1}, \ldots, a_{m}\right)+a_{1} b_{m-1}+\cdots+a_{m} b_{0}}\right| .
$$

Then

$$
\begin{aligned}
\Sigma & :=\sum_{d} 2^{\alpha} 2^{(\alpha+1) m} R_{N}\left(f_{d}\right) \\
& =\sum_{k} \sum_{d}\left|\sum_{a_{1}, \ldots, a_{m}=0}^{1}(-1)^{d\left(a_{1}, \ldots, a_{m}\right)+a_{1} b_{m-1}+\cdots+a_{m} b_{0}}\right| \\
& =\sum_{k} \sum_{d}\left|\sum_{d_{1}, \ldots, d_{m}=0}^{1}(-1)^{d\left(d_{1}, \ldots, d_{m}\right)}\right| \geq 2 \cdot 2^{m} \sum_{i=0}^{2^{m-1}}\left(\begin{array}{c}
2^{m} \\
i
\end{array}\right)\left(2^{m-1}-i\right) .
\end{aligned}
$$


Because of

$$
\begin{gathered}
\sum_{i=0}^{M}\left(\begin{array}{c}
2 M \\
i
\end{array}\right)(M-i)=M\left(\frac{1}{2} \sum_{i=0}^{2 M}\left(\begin{array}{c}
2 M \\
i
\end{array}\right)+\frac{1}{2}\left(\begin{array}{c}
2 M \\
M
\end{array}\right)\right)-\sum_{i=0}^{M} i\left(\begin{array}{c}
2 M \\
i
\end{array}\right) \\
=\frac{M}{2} 2^{2 M}+\frac{M}{2}\left(\begin{array}{c}
2 M \\
M
\end{array}\right)-2 M \sum_{i=0}^{M-1}\left(\begin{array}{c}
2 M-1 \\
i
\end{array}\right)=\frac{M}{2}\left(\begin{array}{c}
2 M \\
M
\end{array}\right),
\end{gathered}
$$

we have $\Sigma \geq 2^{2 m-1}\left({ }_{2^{m-1}}^{2^{m}}\right)$ and therefore there exists a $d$ such that for the net defined by $\bar{d}$ and the function $f_{d}$ we have

$$
R_{N}\left(f_{d}\right) \geq 2^{-2^{m}-1}\left(\begin{array}{c}
2^{m} \\
2^{m-1}
\end{array}\right) 2^{-\alpha} 2^{-(\alpha-1) m} .
$$

By Stirling's formula we have $2^{-\hat{L} M}\left(\begin{array}{c}2 M \\ M\end{array}\right) \sim \pi^{-1 / 2} M^{-1 / 2}$, and the result follows.

So even with optimal nets, that is, with $(0, m, s)$-nets, the quality of the classical estimates cannot be obtained. But we will show in the following that if we restrict to a certain class of nets, we will be able to obtain, and in a certain sense even to improve, these estimates. Further, we will give some concrete, constructive methods for the generation of such special nets.

\section{INTEGRATION WITH DIGITAL NETS}

The following class of nets was introduced in a slightly more general form by Niederreiter in [14].

Definition. Let $b \geq 2$ be a given base. Let $R:=\{\overline{0}, \ldots, \overline{b-1}\}$ be an arbitrary ring with zero element $\overline{0}$. Let $C_{i}=\left(c_{k l}^{(i)}\right), k, l=1, \ldots, m, i=1, \ldots, s$, be $s$ given $m \times m$-matrices over $R$.

In the following we identify every integer $n, 0 \leq n \leq b^{m}-1, n=a_{m-1} b^{m}+$ $\cdots+a_{0}, a_{i} \in\{0, \ldots, b-1\}$, with the vector $\vec{n}:=\left(\bar{a}_{m-1}, \ldots, \bar{a}_{0}\right)^{T}$ and conversely. Then let

$$
\mathbf{x}_{n}:=\left(\frac{1}{b^{m}}\left(C_{1} \cdot \vec{n}\right), \ldots, \frac{1}{b^{m}}\left(C_{s} \cdot \vec{n}\right)\right) \in[0,1)^{s} .
$$

In [14] conditions were given for the above point set to be a $(t, m, s)$-net to base $b$.

For example, if $b$ is a prime power, $R=F_{b}$ the Galois field of order $b$, and $c_{1}^{(i)}, \ldots, c_{m}^{(i)}$ the row vectors of $C_{i}$, then $\mathbf{x}_{n}, n=0,1, \ldots, b^{m}-1$, is a $(t, m, s)$-net to base $b$ if and only if for all $d_{1}, \ldots, d_{s} \in \mathbb{N} \cup\{0\}$ with $d_{1}+\cdots+d_{s}=m-t$ we have: the set of vectors $\left\{c_{j}^{(i)} \mid j=1, \ldots, d_{i}, i=\right.$ $1, \ldots, s\}$ is linearly independent over $F_{b}$. (See [14, Theorem 6.10, Theorem 6.14].) In the following we will call nets of this form digital nets. (See also [9] and [16].)

The following result of Niederreiter gives concrete examples of such digital nets. (See [14, Theorem 6.2].)

Let $b \geq 2, b=q_{1} \cdots q_{u}$, with pairwise relatively prime prime powers $q_{i}$ with $q_{1}<\cdots<q_{u}, m \in \mathbb{N}$ arbitrary, and $s \leq q_{1}+1$. Let $R$ be the direct product of the $F_{q_{i}}, i=1, \ldots, u$. Then the following matrices $C_{i}, i=1, \ldots, s$, 
provide a $(0, m, s)$-net to base $b$ : for every $v, 1 \leq v \leq u$, we choose $s-1$ pairwise different elements $\beta_{i}^{(v)}, i=1, \ldots, s-1$, from $F_{q_{v}}$. Let $\beta_{i}:=$ $\left(\beta_{i}^{(1)}, \ldots, \beta_{i}^{(u)}\right) \in R, 1 \leq i \leq s-1$. Let $C_{i}:=\left(c_{j r}^{(i)}\right), 1 \leq j \leq m, 0 \leq r \leq$ $m-1$. Let

$$
\begin{aligned}
& c_{j r}^{(i)}=0 \text { for } 1 \leq i \leq s-1,1 \leq j \leq m, 0 \leq r<j-1, \\
& c_{j r}^{(i)}=\left(\begin{array}{c}
r \\
j-1
\end{array}\right) \beta_{i}^{r-j+1} \quad \text { for } 1 \leq i \leq s-1,1 \leq j \leq m, j-1 \leq r \leq m-1, \\
& c_{j r}^{(s)}=\delta_{r, m-j} \text { for } 1 \leq j \leq m, 0 \leq r \leq m-1 .
\end{aligned}
$$

Here, $\beta^{0}=1$ for all $\beta \in R$, and $\delta$ is the Kronecker symbol. (In the following we will call these special digital nets " $N$-nets".) Simple examples of $N$-nets are the following: $s=2, b \geq 2$ :

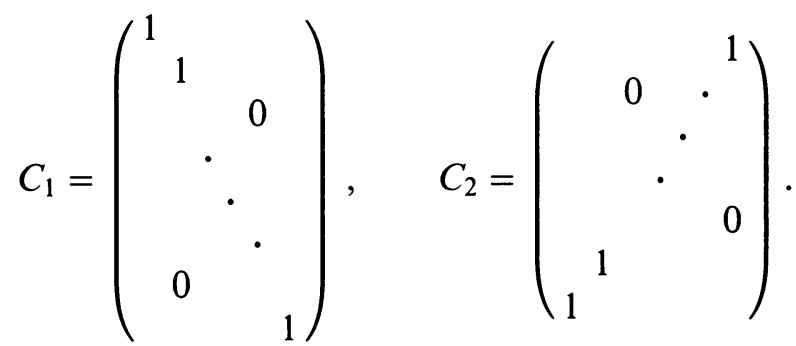

This choice of the $C_{i}$ provides the so-called Hammersley sequence to base $b$, which is well known in uniform distribution. (See [8].)

$s=3, b=2: C_{1}$ like above, $C_{3}=C_{2}$ from above, and

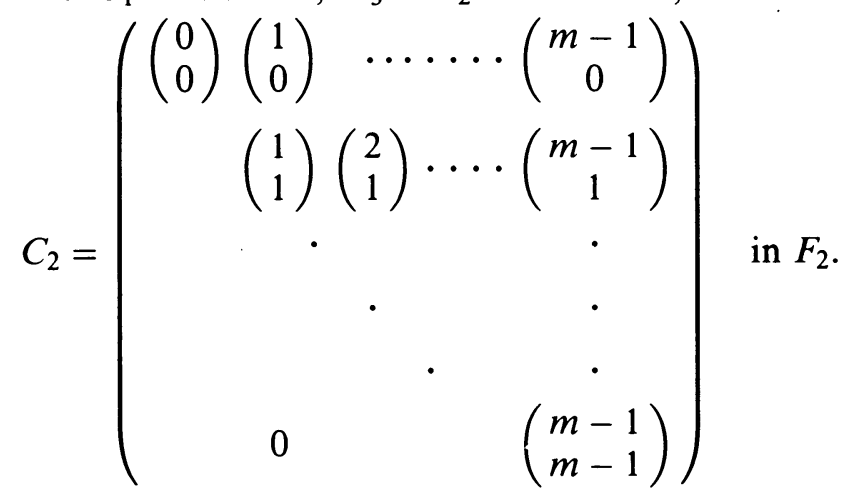

Remark 3. In [14] it was shown that a necessary condition for the existence of a $(0, m, s)$-net to base $b$ is that $s \leq b+1$. On the other hand, the above construction proves the existence of digital $(0, m, s)$-nets to base $b$ whenever $b$ is a prime power with $s \leq b+1$. In the case when $s>b+1$ there remains the question how small $t$ can be such that a $(t, m, s)$-net to base $b$ exists. Quite recently, intensive computer calculations were carried out to solve this question for base $b=2$ and certain values of $m$ and $s$, by Hansen, Mullen, and Niederreiter in [3]. So, for example, they gave concrete examples of $(6,20,4)$ digital nets or of $(6,10,12)$-digital nets. Currently, much research is being done in this direction. As a theoretical result, the following is known from [15]:

For every dimension $s$ and every base $b$ there exists a constant $\tau(s, b)$ such that for all $m \in \mathbb{N}$ there exists a $(t, m, s)$-net to base $b$ with $t \leq \tau(s, b)$. (For similar results for a special class of digital nets see [9].) 
For digital nets and prime base $b$ we now show the following improvement of the estimate of Theorem 1(b):

Theorem 3. For a given prime $b \geq 2$ let $\mathbf{x}_{1}, \ldots, \mathbf{x}_{N}, N=b^{m}$, be a digital $(t, m, s)$-net to base $b$. Let $f \in \bar{E}_{s}^{\alpha}(c)$ to base $b$. Then

$$
R_{N}(f) \leq c^{\prime}(b, s, \alpha, c) b^{t \alpha} \frac{(\log N)^{s-1}}{N^{\alpha}}
$$

Corollary 1. Let $f \in \bar{E}_{s}^{\alpha}(c)$ to prime base $b$. Take for $\mathbf{x}_{1}, \ldots, \mathbf{x}_{N}$ the $(0, m, s)$ $N$-net to base $b \quad\left(N=b^{m}\right)$. Then

$$
R_{N}(f) \leq c^{\prime}(b, s, \alpha, c) \frac{(\log N)^{s-1}}{N^{\alpha}}
$$

Corollary 2. For every dimension $s$, every prime base $b \geq 2$, and every $m \in \mathbb{N}$, there exists a digital net $\mathbf{x}_{1}, \ldots, \mathbf{x}_{N}$ to base $b\left(N=b^{m}\right)$ such that

$$
R_{N}(f) \leq c^{\prime}(b, s, \alpha, c) b^{\tau(s, b) \alpha} \frac{(\log N)^{s-1}}{N^{\alpha}} \text { for all } f \in \bar{E}_{s}^{\alpha}(c)
$$

Remark. In the statement and proof of Theorem 3 we limit ourselves to the case that $b$ is a prime. Although numerical investigations suggest that the assertion of the theorem is probably true also for an arbitrary base, an attempt to prove the theorem in this general case causes technical problems. The main reason for these problems is that Lemma $6(\mathrm{~b})$ is no longer true for arbitrary bases.

In the following let $Z_{b}:=\{\overline{0}, \ldots, \overline{b-1}\}$. Again, we use the identification $n \leftrightarrow \vec{n}$ in the natural way. Further, for $m, n \in \mathbb{N}$ we denote by $m \oplus n$ the integer which corresponds to $\vec{m}+\vec{n}$.

Lemma 6. Let $b, m \in \mathbb{N}, b \geq 2$ be fixed, Let $k, l, n \in \mathbb{N}_{0}$ be given with $0 \leq n<b^{m}$, and let $B=\left(b_{i j}\right)$ be an $m \times m$-matrix over $F_{b}$. Then:

(a) For $x \in[0,1): \operatorname{wal}_{k}(x) \cdot \operatorname{wal}_{l}(x)=\operatorname{wal}_{k \oplus l}(x)$.

(b) $\operatorname{wal}_{k}\left(B \cdot \vec{n} / b^{m}\right)=$ wal $_{B^{*} \cdot \vec{k}\left(\bmod b^{m}\right)}\left(n / b^{m}\right)$, where $B^{*}=\left(b_{m+1-j, m+1-i}\right)$.

Proof. (a) follows immediately from Lemma 5.

(b) Let $\vec{n}=\left(\bar{a}_{m-1}, \ldots, \bar{a}_{0}\right)^{T}$; then

$$
B \cdot \vec{n}=\left(\sum_{i=1}^{m} b_{1 i} \bar{a}_{m-i}, \ldots, \sum_{i=1}^{m} b_{m i} \bar{a}_{m-i}\right) .
$$

In the following we omit the bar - over elements of $F_{b}$. It will be clear in every case whether the digit or the corresponding element of the field is used.

Then, by Lemma 5 with $k=c_{r-1} b^{r-1}+\cdots+c_{0}$ (without loss of generality we can assume $r \geq m$ ) we have

$$
\begin{aligned}
\operatorname{wal}_{k}\left(B \cdot \vec{n} / b^{m}\right)=w^{t} \text { with } t & =\sum_{j=0}^{m-1} c_{j} \sum_{i=1}^{m} b_{j+1, i} a_{m-i} \\
& =\sum_{i=1}^{m} a_{m-i} \sum_{j=0}^{m-1} b_{j+1, i} c_{j}
\end{aligned}
$$


and therefore

$$
\begin{aligned}
w^{t}=\operatorname{wal}_{q}\left(n / b^{m}\right) \quad \text { with } \vec{q} & =\left(\sum_{j=0}^{m-1} b_{j+1, m} c_{j}, \ldots, \sum_{j=0}^{m-1} b_{j+1,1} c_{j}\right) \\
& =B^{*} \cdot \vec{k}\left(\bmod b^{m}\right) .
\end{aligned}
$$

Lemma 7. Let $C_{1}, \ldots, C_{s}$ be $m \times m$-matrices over $F_{b}=Z_{b}$ with row vectors $c_{j}^{(i)}, j=1, \ldots, m, i=1, \ldots, s$, and with the property that for all $d_{1}, \ldots, d_{s} \in \mathbb{N} \cup\{0\}$ with $d_{1}+\cdots+d_{s}=m-t$ the set $\left\{c_{j}^{(i)}, j=1, \ldots, d_{i}\right.$, $i=1, \ldots, s\}$ is linearly independent over $F_{b}$. Let $k_{1}, \ldots, k_{s}$ be integers with $0 \leq k_{i}<b^{m}$, not all zero, and such that $C_{1}^{*} \vec{k}_{1}+\cdots+C_{s}^{*} \vec{k}_{s}=(0, \ldots, 0)^{T}$. Then we have $\prod_{i=1}^{s} \max \left(1, k_{i}\right) \geq b^{m-t-s+1} .\left(C_{i}^{*}\right.$ is defined like $B^{*}$ in Lemma 6(b).) Proof. Let $\vec{k}_{i}=\left(k_{m-1}^{(i)}, \ldots, k_{0}^{(i)}\right)^{T}$; then by insertion we get that the condition $C_{1}^{*} \vec{k}_{1}+\cdots+C_{s}^{*} \vec{k}_{s}=(0, \ldots, 0)^{T}$ is equivalent to $\sum_{i=1}^{s} \sum_{j=0}^{m-1} k_{j}^{(i)} c_{j+1}^{(i)}=$ $(0, \ldots, 0)$. Because of the condition for the $C_{i}$ there must exist $t_{i(1)}, \ldots, t_{i(r)}$ $>0,1 \leq i(1)<i(2)<\cdots<i(r) \leq s$ with $t_{i(1)}+\cdots+t_{i(r)} \geq m-t+1$ and $k_{t_{i(l)}-1}^{(i(l))} \neq 0$ for $l=1, \ldots, r$. Therefore, $k_{i(l)} \geq b^{t_{i(l)}-1}$ and $\prod_{i=1}^{s} \max \left(1, k_{i}\right) \geq$ $b^{m-t-s+1}$.

Proof of Theorem 3. (We follow the ideas of Hlawka in [4] in the classical case. See also [11] and [21].) With $\mathbf{x}_{n}:=\left(C_{1} \cdot \vec{n} / b^{m}, \ldots, C_{s} \cdot \vec{n} / b^{m}\right), n=$ $0, \ldots, b^{m}-1$, we have

$$
\begin{aligned}
R_{N}(f) \leq \frac{c}{N} \sum_{r=1}^{s} \sum_{1 \leq i_{1}<\cdots<i_{r} \leq s} & \sum_{k_{i_{1}}, \ldots, k_{i_{r}}=1}^{\infty}\left(k_{i_{1}} \cdots k_{i_{r}}\right)^{-\alpha} \\
& \times \sum_{n=0}^{N-1} \mathrm{wal}_{k_{i_{1}}}\left(C_{i_{1}} \cdot \vec{n} / b^{m}\right) \cdots \mathrm{wal}_{k_{i_{r}}}\left(C_{i_{r}} \cdot \vec{n} / b^{m}\right) \\
= & \frac{c}{N} \sum_{r} \sum_{i_{j}} \sum_{k_{i_{j}}}\left(k_{i_{1}} \cdots k_{i_{r}}\right)^{-\alpha} \sum_{n=0}^{b^{m}-1} \mathrm{wal}_{C_{i_{1}}^{*} k_{i_{1}}+\cdots+C_{i_{r}}^{*} k_{i_{r}}}\left(n / b^{m}\right)
\end{aligned}
$$

(here we use the notation $\bar{k}=\vec{k}\left(\bmod b^{m}\right)$ and the result of Lemma $6(\mathrm{~b}))$

$$
=c \sum_{r} \sum_{i_{j}} \sum_{\substack{k_{i_{j}} \\ C_{i_{1}}^{*} \bar{k}_{i_{1}}+\cdots+C_{i_{r}}^{*} \bar{k}_{i_{r}}=(0, \ldots, 0)^{T}}}\left(k_{i_{1}} \cdots k_{i_{r}}\right)^{-\alpha} .
$$

Now we consider sums of the form of the inner sum in the last expression:

$$
\sum_{\substack{l_{1}, \ldots, l_{r}=1 \\ B_{1} l_{1}+\cdots+B_{r} l_{r}=(0, \ldots, 0)^{T}}}^{\infty}\left(l_{1} \cdots l_{r}\right)^{-\alpha}=: \Sigma,
$$

where $B_{1}, \ldots, B_{r}$ are such that from $B_{1} \bar{d}_{1}+\cdots+B_{r} \bar{d}_{r}=(0, \ldots, 0)^{T}$ we get $\prod_{i=1}^{r} \max \left(d_{i}, 1\right) \geq b^{A}$ for a certain $A \leq m$. (We denote this condition by $(*)$.) 
If there were nonnegative integers $v, a_{i}$, and $t_{i}<m, i=1, \ldots, r-1$, and nonnegative integers $l_{i}, l_{i}^{\prime}$ with

$$
a_{i} b^{t_{i}} \leq l_{i}, l_{i}^{\prime}<\left(a_{i}+1\right) b^{t_{i}} \text { for } i=1, \ldots, r-1
$$

and

$$
v b^{A-t_{1}-\cdots-t_{r-1}-(r-1)} \leq l_{r}, l_{r}^{\prime}<(v+1) b^{A-t_{1}-\cdots-t_{r-1}-(r-1)}
$$

such that $\left(l_{1}, \ldots, l_{r}\right) \neq\left(l_{l}^{\prime}, \ldots, l_{r}^{\prime}\right)$ and such that

$$
B_{1} \bar{l}_{1}+\cdots+B_{r} \bar{l}_{r}=B_{1} \bar{l}_{1}^{\prime}+\cdots+B_{r} \bar{l}_{r}^{\prime}=(0, \ldots, 0)^{T},
$$

then we would have $\left(\bar{l}_{1}, \ldots, \bar{l}_{r}\right) \neq\left(\bar{l}_{1}^{\prime}, \ldots, \bar{l}_{r}^{\prime}\right)$, and with

$$
\begin{aligned}
& L_{i}:=l_{i}^{\prime} \ominus l_{i}<b^{t_{i}} \text { for } i=1, \ldots, r-1, \\
& L_{r}:=l_{r}^{\prime} \ominus l_{r}<b^{A-t_{1}-\cdots-t_{r-1}-(r-1)}
\end{aligned}
$$

we would have a further solution of $B_{1} \bar{L}_{1}+\cdots+B_{r} \bar{L}_{r}=(0, \ldots, 0)^{T}$ for which $\left(\bar{L}_{1}, \ldots, \bar{L}_{r}\right) \neq(0, \ldots, 0)$ and where $\prod_{i=1}^{r} \max \left(1, L_{i}\right)<b^{A-(r-1)}$, which is a contradiction. We denote this fact by $(* *)$.

So we have

$$
\begin{aligned}
\Sigma \leq & \sum_{\substack{l_{1}, \ldots, l_{r}=1 \\
B_{1} l_{1}+\cdots+B_{r} l_{r}=(0, \ldots, 0)^{T}}}^{b^{m}-1}\left(l_{1} \ldots l_{r}\right)^{-\alpha} \\
& +\sum_{t=1}^{r} \sum_{l_{1}, \ldots, l_{t-1}, l_{t+1}, \ldots, l_{r}=1}^{\infty}\left(l_{1} \cdots l_{t-1} l_{t+1} \cdots l_{r}\right)^{-\alpha} \sum_{\substack{l_{l}=b^{m} \\
B_{1} l_{1}+\cdots+B_{r} l_{r}=(0, \ldots, 0)^{T}}}^{\infty} l_{t}^{-\alpha} \\
=: & \Sigma_{1}+\Sigma_{2} .
\end{aligned}
$$

Now because of $(*)$ :

$$
\begin{aligned}
& \Sigma_{1} \leq \sum_{t_{1}=0}^{m-1} \sum_{a_{1}=1}^{b-1} \sum_{l_{1}=a_{1} b^{t_{1}}}^{\left(a_{1}+1\right) b_{1}^{t_{1}}} l_{1}^{-\alpha} \ldots \sum_{t_{r-1}=0}^{m-1} \sum_{a_{r-1}=1}^{b-1} \sum_{l_{r-1}=a_{r-1} b^{t_{r-1}}}^{\left(a_{r-1}+1\right) b^{t_{r}-1}} l_{r-1}^{-\alpha} \\
& \times \sum_{v=1}^{\infty} \sum_{l_{r}=v b^{A-t_{1}}-\cdots-t_{r-1}-(r-1)}^{(v+1) b^{A-t_{1}-\cdots-t_{r-1}-(r-1)}-1} l_{r}^{-\alpha}
\end{aligned}
$$

(where summation is over all $l_{1}, \ldots, l_{r}$ with $\left.B_{1} \bar{l}_{1}+\cdots+B_{r} \bar{l}_{r}=(0, \ldots, 0)^{T}\right)$ and this, because of $(* *)$, is at most

$$
\begin{gathered}
\sum_{t_{1}=0}^{m-1} \sum_{a_{1}=1}^{b-1}\left(a_{1} b^{t_{1}}\right)^{-\alpha} \ldots \sum_{t_{r-1}=0}^{m-1} \sum_{a_{r-1}=1}^{b-1}\left(a_{r-1} b^{t_{r-1}}\right)^{-\alpha} \\
\times \sum_{v=1}^{\infty}\left(v b^{A-t_{1}-\cdots-t_{r-1}-(r-1)}\right)^{-\alpha} \\
\leq b^{(r-1) \alpha} \frac{1}{b^{A \alpha}} m^{r-1} \zeta^{r}(\alpha) .
\end{gathered}
$$


For $\Sigma_{2}$ we have (by taking in $(* *) t_{1}=t_{2}=\cdots=t_{r-1}=0$ and because of $A \leq m)$

$$
\begin{aligned}
\Sigma_{2} & \leq r \sum_{l_{1}, \ldots, l_{r-1}=1}^{\infty}\left(l_{1} \cdots l_{r-1}\right)^{-\alpha} \sum_{v=1}^{\infty}\left(v b^{A-(r-1)}\right)^{-\alpha} \\
& \leq r b^{(r-1) \alpha} \frac{1}{b^{A \alpha}}(\zeta(\alpha))^{r} .
\end{aligned}
$$

Altogether, with $A=m-t-s+1$, we obtain

$$
R_{N}(f) \leq c \sum_{r=1}^{s}\left(\begin{array}{l}
s \\
r
\end{array}\right)\left(\Sigma_{1}+\Sigma_{2}\right) \leq c^{\prime}(b, s, \alpha, c) \frac{(\log N)^{s-1}}{N^{\alpha}} b^{t \alpha} .
$$

For nets with $t=0$ we thus have a maximal integration error of the form $(\log N)^{s-1} / N^{\alpha}$. We will now show that this is the best possible result for the numerical integration of $\bar{E}_{s}^{\alpha}$-functions in any case.

Theorem 4. For all $b \geq 2, c>0, \alpha>1, s \in \mathbb{N}$ there exist constants $c^{\prime}(c, \alpha, s, b)>0$ such that: For all $N$ and every point set $\mathbf{x}_{k}, k=1, \ldots, N$, in $[0,1)^{s}$, there is a $f \in \bar{E}_{s}^{\alpha}(c)$ with $f\left(\mathbf{x}_{k}\right)=0$ for $k=1, \ldots, N$ and

$$
\int_{[0,1)^{s}} f(\mathbf{x}) d \mathbf{x} \geq c^{\prime} \frac{(\log N)^{s-1}}{N^{\alpha}} .
$$

Remark. An analog to this theorem for the class $E_{s}^{\alpha}$ was shown by Šarygin [18]. (See also [5].)

Proof of Theorem 4. Let $t$ be such that $b^{t-1} \leq N<b^{t}$. We add $b^{t}-N-1$ points to the initial point set and denote the new set by $\mathbf{x}_{1}, \ldots, \mathbf{x}_{b^{t}-1}$. For integer-valued vectors $\mathbf{r}:=\left(r_{1}, \ldots, r_{s}\right), r_{1}+\cdots+r_{s}=t, r_{i} \geq 0$, we consider the set $M(\mathbf{r})$ of all integer-valued vectors $\left(m_{1}, \ldots, m_{s}\right), m_{i} \geq 0$, with $m_{i}<b^{r_{i}}$ for all $i=1, \ldots, s . M(\mathbf{r})$ has $b^{t}$ elements. Therefore, the linear system

$$
\sum_{\mathbf{m} \in M(\mathbf{r})} c_{\mathbf{r}}(\mathbf{m}) \operatorname{wal}_{\mathbf{m}}\left(\mathbf{x}_{k}\right)=0, \quad k=1, \ldots, b^{t}-1,
$$

for every $\mathbf{r}$ has a nontrivial solution $c_{\mathbf{r}}(\mathbf{m}), \mathbf{m} \in M(\mathbf{r})$. Let $\left|c_{\mathbf{r}}\left(\mathbf{m}^{\prime}(\mathbf{r})\right)\right|:=$ $\max _{\mathbf{m} \in M(\mathbf{r})}\left|c_{\mathbf{r}}(\mathbf{m})\right|, \mathbf{m}^{\prime \prime}(\mathbf{r})$ the unique element in $M(\mathbf{r})$ with $\mathbf{m}^{\prime}(\mathbf{r}) \oplus \mathbf{m}^{\prime \prime}(\mathbf{r})=\mathbf{0}$, and

$$
W_{\mathbf{r}}(\mathbf{x}):=\frac{\mathrm{wal}_{\mathbf{m}^{\prime \prime}(\mathbf{r})}(x)}{c_{\mathbf{r}}\left(\mathbf{m}^{\prime}(\mathbf{r})\right) b^{\alpha t}} \sum_{\mathbf{m} \in M(\mathbf{r})} c_{\mathbf{r}}(\mathbf{m}) \mathrm{wal}_{\mathbf{m}}(\mathbf{x})
$$

We have $W_{\mathbf{r}} \not \equiv 0$. Let

$$
f(\mathbf{x}):=c \frac{1}{C^{\prime}} \sum_{\mathbf{r}} W_{\mathbf{r}}(\mathbf{x}) \quad \text { with } C^{\prime}:=\sup _{0 \leq y \leq 1} y^{\alpha}\left(1-\frac{\log y}{\log b}\right)^{s}, C^{\prime}<\infty .
$$

We show $f \in \bar{E}_{s}^{\alpha}(c)$ : For given $\overline{\mathbf{m}}$, the term wal $_{\mathbf{m}}$ occurs in $W_{\mathbf{r}}$ if and only if there exists an $\mathbf{m} \in M(\mathbf{r})$ with $\mathbf{m} \oplus \mathbf{m}^{\prime \prime}(\mathbf{r})=\overline{\mathbf{m}}$. This is possible only if $\overline{\mathbf{m}} \in M(\mathbf{r})$. In this case there exists exactly one such $\mathbf{m}$. Therefore, for the $\overline{\mathbf{m}}$ th Walsh coefficient $\bar{f}(\overline{\mathbf{m}})$ of $f$ we have

$$
|\bar{f}(\overline{\mathbf{m}})| \leq c \frac{1}{C^{\prime}} b^{-\alpha t} \sum_{\substack{\mathbf{r} \\ \mathbf{m} \in M(\mathbf{r})}} 1 .
$$


From $\overline{\mathbf{m}} \in M(\mathbf{r})$ we have $r_{i} \geq{ }^{b} \log \bar{m}_{i}, i=1, \ldots, s$, and therefore ${ }^{b} \log \bar{m}_{i} \leq r_{i}=t-r_{1}-\cdots-r_{i-1}-r_{i+1}-\cdots-r_{s} \leq{ }^{b} \log \left(\frac{b^{t}}{\bar{m}_{1} \cdots \bar{m}_{i-1} \bar{m}_{i+1} \cdots \bar{m}_{s}}\right)$, $\overline{\mathbf{m}}:=\left(m_{1}, \ldots, m_{s}\right)$ and $\left.\bar{m}_{i}:=\max \left(1, m_{i}\right)\right)$. Therefore,

$$
\begin{aligned}
\mid \bar{f}(\overline{\mathbf{m}}) & \leq c \frac{1}{C^{\prime}} \frac{\left(\bar{m}_{1} \cdots \bar{m}_{s}\right)^{\alpha}}{b^{\alpha t}}\left({ }^{b} \log \frac{b^{t}}{\bar{m}_{1} \cdots \bar{m}_{s}}+1\right)^{s}\left(\bar{m}_{1} \cdots \bar{m}_{s}\right)^{-\alpha} \\
& \leq c\left(\bar{m}_{1} \cdots \bar{m}_{s}\right)^{-\alpha} .
\end{aligned}
$$

So, $f \in \bar{E}_{s}^{\alpha}(c), f\left(\mathbf{x}_{k}\right)=0, k=1, \ldots, N$, and

$$
\begin{aligned}
\int_{[0,1)^{s}} f(\mathbf{x}) d \mathbf{x} & =c \frac{1}{C^{\prime}} b^{-\alpha t} \sum_{\mathbf{r}} \int_{[0,1)^{s}} \sum_{\mathbf{m} \in M(\mathbf{r})} \frac{C_{\mathbf{r}}(\mathbf{m})}{C_{\mathbf{r}}\left(\mathbf{m}^{\prime}(\mathbf{r})\right)} \operatorname{wal}_{\mathbf{m}^{\prime \prime}(\mathbf{r}) \oplus \mathbf{m}}(\mathbf{x}) d \mathbf{x} \\
& =c \frac{1}{C} b^{-\alpha t} \sum_{\mathbf{r}} \int_{[0,1)^{s}} 1 d \mathbf{x}=c \frac{1}{C^{\prime}} b^{-\alpha t} \sum_{r_{1}+\cdots+r_{s}=t} 1 \\
& \geq C^{\prime \prime} \frac{(\log N)^{s-1}}{N^{\alpha}} .
\end{aligned}
$$

Digital nets, for example digital $N$-nets, therefore provide best possible, and, as will be shown in the concluding section, good programmable methods for the numerical integration of Walsh series.

\section{EXAMPLES}

Example 1. Let $e=0 . e_{1} e_{2} e_{3} \ldots, e_{i} \in\{0,1\}, i=1,2, \ldots$, be a fixed given 0,1 -signal, considered as a real number in $[0,1]$ represented to base 2. The function $f_{e}:[0,1]^{2} \rightarrow[0,1]$ bundles two incoming 0,1 -signals $x=$ $0 . x_{1} x_{2} \ldots$ and $y=0 . y_{1} y_{2} \ldots$ and the given $e$ to the new signal $f_{e}(x, y):=$ $0 . x_{1} y_{1} e_{1} x_{2} y_{2} e_{2} \ldots$ represented again as a real number to base 2 . It is easy to show that

$$
\begin{aligned}
f_{e}(x, y)= & \frac{1}{2}+\sum_{l=1}^{\infty} 2^{-3 l-1}(-1)^{\left[2^{l} e\right]+1} \\
& +\sum_{l=1}^{\infty}\left(\left(-2^{-3 l+1}\right)(-1)^{\left[2^{l} x\right]}+\left(-2^{-3 l}\right)(-1)^{\left[2^{l} y\right]},\right.
\end{aligned}
$$

and therefore, $f_{e} \in \bar{E}_{2}^{3}(2)$. For concrete values of $e$ we get, for example,

$$
\int_{[0,1)^{2}} f_{0}=3 / 7=0 . \overline{428571}, \quad \int_{[0,1)^{2}} f_{1 / 2}=31 / 56=0.553 \overline{571428} .
$$

Numerical integration with the uniform lattice, respectively with the Hammersley sequence (digital $N$-net) to base 2 ( $N$ points in each case) gives the following results: 
TABLE 1. Results for $e=0$

\begin{tabular}{|c|c|c|c|c|c|}
\hline$m$ & $N$ & unif. lattice & $R_{N} \sim$ & Hammersley seq. & $R_{N} \sim$ \\
\hline 4 & 16 & 0.421875 & $10^{-3}$ & 0.428466796875 & $10^{-4}$ \\
\hline 6 & 64 & 0.427734375 & $10^{-3}$ & 0.428569793701172 & $10^{-5}$ \\
\hline 8 & 256 & 0.428466796875 & $10^{-4}$ & 0.428571403026581 & $10^{-8}$ \\
\hline 10 & 1024 & 0.4285583496093375 & $10^{-5}$ & 0.42857142817229 & $10^{-10}$ \\
\hline 12 & 4096 & 0.428569793701172 & $10^{-5}$ & 0.428571428565192 & $10^{-11}$ \\
\hline 14 & 16384 & 0.428571224212646 & $10^{-7}$ & 0.428571428571331 & $10^{-13}$ \\
\hline
\end{tabular}

TABLE 2. Results for $e=1 / 2$

\begin{tabular}{rrllll}
$m$ & $N$ & \multicolumn{1}{c}{ unif. lattice } & $R_{N} \sim$ & Hammersley seq. & $R_{N} \sim$ \\
& & & & & \\
6 & 64 & 0.552734375 & $10^{-3}$ & 0.553569793701172 & $10^{-5}$ \\
10 & 1024 & 0.553558349609375 & $10^{-5}$ & 0.55357142817229 & $10^{-10}$ \\
14 & 16384 & 0.553571224212646 & $10^{-7}$ & 0.553571428571331 & $10^{-13}$
\end{tabular}

Example 2. Let $f:[0,1)^{3} \rightarrow \mathbb{R}$ be given by $f(x, y, z)=g(x) g(y) g(z)$ with $g:[0,1) \rightarrow \mathbb{R}, g(x)=2^{-l-1}$ for $2^{-l-1} \leq x<2^{-l}, l=0,1, \ldots$. As is easily checked, we have

$$
g(x)=\frac{1}{6}\left(2-\sum_{k=0}^{\infty} 2^{-2 k} \sum_{l=2^{k}}^{2^{k+1}-1} \mathrm{wal}_{l}(x)\right) \text { and } \int_{0}^{1} g(x) d x=\frac{1}{3} .
$$

Therefore, $f \in \bar{E}_{3}^{2}(1)$ and $\int_{[0,1]^{3}} f(\mathbf{x}) d \mathbf{x}=\frac{1}{27}=0 . \overline{037}$. Numerical integration with the uniform lattice, respectively with the three-dimensional $N$-net, given as an example in $\S 3$, gives:

TABLE 3

\begin{tabular}{rrcccc}
$m$ & \multicolumn{1}{c}{$N$} & unif. lattice & $R_{N} \sim$ & $N$-net & $R_{N} \sim$ \\
& & & & & \\
6 & 64 & 0.030517578125 & $10^{-2}$ & 0.036239624023438 & $10^{-3}$ \\
9 & 512 & 0.035327911376953 & $10^{-3}$ & 0.036406806945801 & $10^{-4}$ \\
12 & 4096 & 0.036604702472687 & $10^{-4}$ & 0.037025457248092 & $10^{-5}$ \\
15 & 32768 & 0.036928636021912 & $10^{-4}$ & 0.037037021918877 & $10^{-8}$
\end{tabular}

\section{BIBLIOGRAPHY}

1. N. S. Bahvalov, Approximate computation of multiple integrals, Vestnik. Moskov. Univ. Ser. I Mat. Mekh. Astronom. Fiz. Khim. 4 (1959), 3-18. (Russian)

2. H. E. Chrestenson, A class of generalized Walsh functions, Pacific J. Math. 5 (1955), 17-31.

3. T. Hansen, G. L. Mullen, and H. Niederreiter, Good parameters for a class of node sets in quasi-Monte Carlo integration, Math. Comp. 61 (1993), 225-234.

4. E. Hlawka, Zur angenäherten Berechnung mehrfacher Integrale, Monatsh. Math. 66 (1962), 140-151. 
5. L. K. Hua and Y. Wang, Applications of number theory to numerical analysis, SpringerVerlag, Berlin, 1981.

6. N. M. Korobov, The approximate computation of multiple integrals, Dokl. Akad. Nauk SSSR 124 (1959), 1207-1210. (Russian)

7. _ Number-theoretical methods in approximate analysis, Fizmatgiz. Moscow, 1963. (Russian)

8. L. Kuipers and H. Niederreiter, Uniform distribution of sequences, Wiley, New York, 1974.

9. G. Larcher, Nets obtained from rational functions over finite fields, Acta Arith. 63 (1993), 1-13.

10. K. Niederdrenk, Die endliche Fourier- und Walshtransformation mit einer Einführung in die Bildverarbeitung, Vieweg, Braunschweig, 1982.

11. H. Niederreiter, Pseudo-random-numbers and optimal coefficients, Adv. Math. 26 (1977), 99-181.

12. __ Existence of good lattice points in the sense of Hlawka, Monatsh. Math. 86 (1978), 203-219.

13. __ Dyadic fractions with small partial quotients, Monatsh. Math. 101 (1986), 309-315.

14. $\ldots$, Point sets and sequences with small discrepancy, Monatsh. Math. 104 (1987), 273337.

15. __, Low-discrepancy and low-dispersion sequences, J. Number Theory 30 (1988), 51-70.

16. _ Low-discrepancy point sets obtained by digital constructions over finite fields, Czechoslovak Math. J. 42 (1992), 143-166.

17. __ Existence theorems for efficient lattice rules, Numerical Integration: Recent Developments, Software and Applications (T. O. Espelid and A. Genz, eds.), Kluwer, Dordrecht, 1992, pp. 1-80.

18. I. F. Šarygin, $A$ lower estimate for the error of quadrature formulas for certain classes of functions, Ž. Vyčisl. Mat. i Mat. Fiz. 3 (1963), 370-376. (Russian)

19. I. M. Sobol', The distribution of points in a cube and the approximate evaluation of integrals, Ž. Vyčisl. Mat. i Mat. Fiz. 7 (1967), 784-802. (Russian)

20. _ Multidimensional quadrature formulas and Haar functions, Nauka, Moscow, 1969. (Russian)

21. S. K. Zaremba, La méthode des "bon treillis" pour le calcul numérique des integrals multiples, Applications of Number Theory to Numerical Analysis (S. K. Zaremba, ed.), Academic Press, New York, 1972, pp. 39-119.

Institut für Mathematik, Universität Salzburg, Hellbrunnerstrasse 34, A-5020 SAlzburg, Austria

Siemens AG Österreich, System- Und Programmentwicklung, Petersbrunnstrasse 19, A-5020 Salzburg, Austria 